\title{
82. NOVEDADES SINTAXONÓMICAS Y NOMENCLATURALES EN MATORRALES SERIALES VALENCIANOS DEL ORDEN ROSMARINETALIA BR.-BL. EX MOLINIER 1934
}

Manuel B. CRESPO

Syntaxonomical and nomenclatural novelties in shrubland vegetation of Rosmarinetalia Br.-Bl. ex Molinier 1934 from eastern Spain.

Palabras clave. Sintaxonomía, nomenclatura, Rosmarinion, Hypericion, Satureja, Comunidad Valenciana, España.

Key Words. Syntaxonomy, nomenclature, Rosmarinion, Hypericion, Satureja, Valencian Community, eastern Spain.

Los matorrales seriales valencianos se vienen estudiando ampliamente desde antiguo. Particularmente, las comunidades calcícolas de Rosmarinetalia se han tratado en trabajos recientes (cf. Costa et al., 1983; Costa y Peris, 1985; Alcaraz y De la Torre, 1988; De la Torre y Alcaraz, 1994; Stübing et al., 1989), completando la sintaxonomía de este orden en los territorios iberolevantinos.

La revisión que sobre la clase Rosmarinetea han realizado Díez Garretas et al. (1998) ha clarificado aspectos nomenclaturales de gran importancia y ha permitido, asimismo, disponer de una síntesis actualizada de la dispersa información existente sobre este tipo de vegetación en la Península Ibérica y Baleares.

Es bien sabido que la importancia de los matorrales radica en el hecho de que son muy numerosos los endemismos que encuentran sus hábitats en las formaciones heliófilas calcícolas, por lo que este tipo de vegetación presenta un enorme valor desde una óptica conservacionista. La reciente publicación del libro rojo de la flora endémica de la Comunidad Valenciana (Laguna et al., 1998) ha permitido recopilar nuevos datos sobre la distribución de táxones hasta ahora mal conocidos 0 minusvalorados, pero de enorme valor corológico y, en consecuencia, de gran importancia para el análisis de los matorrales valencianos.

Parte de los resultados que se presentan fueron dados a conocer hace algunos años en publicación de tipo microficha (Crespo, 1989), pero dado que el Código de Nomenclatura Fitosociológica (C.N.F.), en su Art. 1, deja abierta la posibilidad de considerar sin valor a efectos nomenclaturales dichas publicaciones (cf. Díez Garretas et al., 1995), preferimos aportar ahora nuevos datos que completan o matizan lo que entonces dijimos. No obstante, sería deseable la modificación de dicho artículo indicando explícitamente qué tipo de material impreso debe considerarse efectivamente publicado, puesto que en su redacción actual difícilmente pueden considerarse las microfichas algo distinto a "material impreso" sobre acetato (en ningún momento se señala en el Código que el soporte sobre el que se imprima sea necesariamente papel).

Sobre la base de los estudios y síntesis aludidos, en el presente trabajo se aportan nuevos datos sobre los matorrales valencianos, con especial hincapié en algunas asociaciones valenciano-castellonenses inéditas o poco conocidas.

Para la terminología bioclimática se siguen las directrices de Rivas Martínez (1987, 1993, 1997) para la Región Mediterránea, mientras que los aspectos corológicos se ajustan a las propuestas de Rivas Martínez (1987) para 
Tabla 1.

\begin{tabular}{|c|c|c|c|c|c|c|c|c|c|c|}
\hline \multirow[b]{2}{*}{$\mathrm{N}^{0}$ de orden } & \multicolumn{10}{|c|}{$\begin{array}{l}\text { Teucrio edetani-Anthyllidetum terniflorae } \mathrm{M} \text {. B. Crespo, ass. nov. } \\
\quad \text { (Rosmarinion officinalis, Rosmarinetalia, Rosmarinetea) }\end{array}$} \\
\hline & 1 & 2 & 3 & 4 & 5 & 6 & 7 & 8 & 9 & 10 \\
\hline Área $\left(\mathrm{m}^{2}\right)$ & 100 & 50 & 50 & 50 & 50 & 50 & 25 & 50 & 50 & 50 \\
\hline Altitud (m.s.n.m.) & 150 & 100 & 180 & 80 & 100 & 150 & 100 & 200 & 200 & 90 \\
\hline Cobertura $(\%)$ & 80 & 85 & 80 & 90 & 80 & 80 & 80 & 85 & 80 & 80 \\
\hline Exposición $\left({ }^{\circ}\right)$ & $20 \mathrm{~S}$ & $10 \mathrm{~S}$ & $20 \mathrm{E}$ & $5 \mathrm{NE}$ & $10 \mathrm{~S}$ & $25 \mathrm{E}$ & - & $10 \mathrm{~S}$ & 10SE & - \\
\hline Fecha (mes-año) & $4-91$ & $5-91$ & $6-93$ & $5-92$ & $6-87$ & $5-92$ & $4-91$ & $4-91$ & $5-92$ & $5-88$ \\
\hline \multicolumn{11}{|l|}{ Caract. asociación y alianza: } \\
\hline Anthyllis terniflora & 3.3 & 2.3 & 2.2 & 3.3 & 2.2 & 2.2 & 2.3 & 3.3 & 2.2 & 2.2 \\
\hline Erica multiflora & 2.2 & 1.2 & 2.2 & 1.2 & 1.1 & 1.2 & +.2 & +.2 & 2.2 & +.2 \\
\hline Sideritis juryi & +.2 & 1.1 & 1.1 & & 1.2 & 1.2 & +.2 & +.2 & 1.1 & +.2 \\
\hline Thymelaea argentata & +.2 & 1.2 & +.2 & 1.1 & +.2 & & +.2 & 1.2 & 1.1 & +.2 \\
\hline Teucrium edetanum & 1.1 & 1.2 & 1.1 & & 1.2 & 1.1 & 1.1 & 1.2 & 1.1 & 1.2 \\
\hline Ulex parviflorus & 1.1 & +.2 & 1.2 & 1.2 & 2.2 & . & & 1.2 & . & +.2 \\
\hline Helianthemum marifolium & & 1.1 & . & +.2 & +.2 & . & 1.1 & & . & +.2 \\
\hline Onobrychis stenorhiza & +.2 & . & . & . & & & . & +.2 & . & . \\
\hline Anthyllis $\times$ media & & . & . & & & . & . & +.2 & +.2 & . \\
\hline Cistus carthaginensis & +.2 & . & . & & & . & . & . & . & . \\
\hline \multicolumn{11}{|l|}{ Diferenciales de variantes: } \\
\hline Helianthemum glabratum & . & . & . & . & & . & . & 1.1 & + & \\
\hline Anthyllis lagascana & . & . & . & . & & . & . & . & . & 1.2 \\
\hline Thymus piperella & . & . & . & . & . & & . & . & & +.2 \\
\hline \multicolumn{11}{|l|}{ Caract. orden y clase: } \\
\hline Rosmarinus officinalis & 2.2 & 2.2 & 2.2 & 3.3 & 2.2 & 2.2 & 2.2 & 2.2 & 2.2 & 1.2 \\
\hline Hippocrepis scorpioides & 1.1 & 1.1 & 1.1 & 1.1 & 1.2 & 1.1 & 1.2 & 1.1 & 1.1 & +.2 \\
\hline Helianthemum syriacum & 1.1 & +.2 & +.2 & + & 1.1 & + & +.2 & +.2 & + & + \\
\hline Teucrium gracillimum & +.2 & 1.2 & 1.2 & 1.1 & 1.1 & +.2 & +.2 & 1.1 & 1.1 & +.2 \\
\hline Helichrysum stoechas & +.2 & +.2 & + & + & 1.2 & . & 1.1 & 1.1 & + & +.2 \\
\hline Fumana thymifolia & 1.1 & +.2 & 1.1 & 1.1 & 1.2 & + & +.2 & 1.1 & & + \\
\hline Fumana spachii & 1.1 & & +.2 & +.2 & +.2 & 1.1 & +.2 & +.2 & +2 & +.2 \\
\hline Fumana ericoides & 1.1 & +.2 & 1.2 & 1.1 & +.2 & 1.1 & . & & 1.1 & +.2 \\
\hline Thymus vulgaris & 1.1 & 1.1 & & +.2 & 1.1 & +.2 & ${ }^{\circ}$ & +.2 & +.2 & +.2 \\
\hline Convolvulus lanuginosus & + & 1.1 & + & & +.2 & + & +.2 & +.2 & 1.1 & . \\
\hline Globularia alypum & +.2 & +.2 & 1.2 & 1.1 & & . & +.2 & 1.1 & 1.2 & \\
\hline Phagnalon rupestre & +.2 & +.2 & + & +.2 & 1.1 & + & . & + & . & \\
\hline Coris fontqueri & + & & +.2 & & +.2 & + & + & +.2 & . & +.2 \\
\hline Viola arborescens & & +2 & +.2 & +2 & +.2 & +.2 & . & + & + & . \\
\hline Helianthemum pilosum & +.2 & 1.1 & & +.2 & +.2 & + & & & & + \\
\hline Dorycnium pentaphyllum & & & +.2 & & & . & +2 & 1.1 & 1.1 & +.2 \\
\hline Ruta angustifolia & +.2 & 1.2 & & & +.2 & & +.2 & +.2 & . & \\
\hline Haplophyllum rosmarinifolium & . & 1.2 & +.2 & i & +.2 & 1.1 & & +.2 & . & \\
\hline Bupleurum fruticescens & + & . & . & & & +.2 & +.2 & . & 1.1 & +.2 \\
\hline Matthiola fruticulosa & +.2 & + & & & + & + & & & . & + \\
\hline Fumana hispidula & +.2 & & & & . & . & 1.2 & 1.1 & . & +.2 \\
\hline Argyrolobium zanonii & + & +.2 & + & & + & . & . & . & . & \\
\hline Cistus clusii & & . & . & 1.2 & 1.1 & . & +.2 & . & $\therefore$ & \\
\hline Coronilla clusii & . & . & . & . & . & . & + & . & 1.1 & +.2 \\
\hline Anthyllis cytisoides & . & . & . & & . & . & . & +.2 & +.2 & + \\
\hline Compañeras: & & & & & & & & & & \\
\hline Brachypodium retusum & 1.2 & 1.2 & 2.2 & 2.3 & 1.1 & 1.2 & 2.2 & 2.2 & 1.2 & 2.2 \\
\hline Asparagus horridus & + & +.2 & +.2 & +.2 & +.2 & + & +.2 & . & +.2 & + \\
\hline Sedum sediforine & + & & + & +.2 & +.2 & + & +.2 & +.2 & +.2 & +.2 \\
\hline Centaurium barrelieri & + & +.2 & + & & +.2 & . & + & + & & \\
\hline Stipa offneri & 2.2 & 2.2 & 1.1 & 1.1 & . & & & 1.1 & 1.1 & 1.1 \\
\hline Atractylis humilis & + & . & + & +.2 & + & . & +.2 & + & . & + \\
\hline Asphodelus ramosus & 1.1 & + & + & + & & . & + & +.2 & +.2 & . \\
\hline Chamaerops humilis & +.2 & & +.2 & & . & . & +.2 & 1.2 & . & + \\
\hline Asperula scabra & + & +.2 & + & + & . & . & +.2 & . & . & \\
\hline Euphorbia serrata & + & . & . & . & + & + & + & . & . & . \\
\hline Pinus halepensis & . & . & . & . & . & +.2 & 2.2 & +.2 & . & \\
\hline Allium moschatum & + & . & . & + & + & . & + & . & . & $\therefore$ \\
\hline Stipa parviflora & +.2 & . & . & & 1.1 & . & . & . & . & 1.1 \\
\hline
\end{tabular}

Otros táxones: Ononis minutissima + en 1, 5 y 7; Launaea fragilis + en 5, 6 y 8; Thesium divaricatum + en 1, 7 y 8; Polygala rupestris + en 1, 7 y 9; Gladiolus illyricum + en 1, 6 y 10; Lapiedra martinezii + en 3,7 y 8 ; Koeleria vallesiana + en 1 y $2 ;$ Avenula bromoides + en 2 y 6; Stipa tenacissima + en 7 y 8; Juniperus oxycedrus +.2 en 1 y 4 ; Ophrys fusca +.2 en 6 y 7 ; Lithodora fruticosa +.2 en 6 y 7; Hypericum ericoides + en 1 y 3; Plantago albicans + en 1 y $6 ;$ Hedysarum spinosissimum + en 1 y $7 ;$ Hedysarum confertum + en 2 y 5; Dipcadi serotinum + en 6 y 7; Aphyllanthes monspeliensis 1.1 en 1; Thymelaea tinctoria +.2 en 3 , Schoenus nigricans, +.2, Rhamnus angustifolia +.2 , en 3 ; Eryngium campestre, +.2 en $9 ;$ Convolvulus althaeoides,,+ Heteropogon contortus + , Sonchus tenerrimus, + en 1; Aristolochia pistolochia, + en 3; Thesium humile, + , Reseda phyteuma, + en 8.

Procedencia de los inventarios: 1.- V: La Pobla de Vallbona, Lloma Llarga, YJ1289. 2.- V: Paterna, YJ1979. 3.- V: La Pobla de Vallbona, El Pelat, YJ1188. 4.- V: Godella, Campo Olivar, YJ2179. 5.- V: Godella, pr. Huerto de San Mauro, YJ2081. 6.- V: Bétera, YJ2089. 7.- V: Paterna, pr. Fuente del Jarro, YJ1478. 8.- V: Serra, Sanatorio de Porta-Coeli, YJ1792. 9.- V: Olocau, Catxirulo de Turó, YJ1293. 10.- V: Paterna, La Cañada, Martinot, YJ1478. 
Tabla 2.

Helianthemo glabrati-Hypericetum ericoidis M. B. Crespo, ass. nov. (Hypericion ericoidis, Rosmarinetalia officinalis, Rosmarinetea)

\begin{tabular}{|c|c|c|c|c|c|c|c|c|c|c|}
\hline $\mathbb{N}^{0}$ de Orden & 1 & 2 & 3 & 4 & 5 & 6 & 7 & 8 & 9 & 10 \\
\hline Área $\left(\mathrm{m}^{2}\right)$ & 30 & 10 & 20 & 15 & 20 & 20 & 15 & 10 & 15 & 20 \\
\hline Cobertura (\%) & 40 & 50 & 30 & 40 & 50 & 40 & 40 & 45 & 30 & 50 \\
\hline Inclinación $\left({ }^{\circ}\right)$ & 30SE & $45 \mathrm{SW}$ & $5 \mathrm{NE}$ & $5 \mathrm{~S}$ & $5 \mathrm{SE}$ & $5 \mathrm{SE}$ & $5 \mathrm{SW}$ & $5 \mathrm{~W}$ & $25 \mathrm{SW}$ & $\checkmark 10 \mathrm{~S}$ \\
\hline Altitud (m.s.n.m.) & 450 & 600 & 280 & 300 & 750 & 350 & 400 & 560 & 150 & 100 \\
\hline Fecha (mes-año) & $8-87$ & $7-91$ & $8-90$ & $8-90$ & $7-91$ & $8-90$ & $7-91$ & $7-91$ & $8-90$ & $7-91$ \\
\hline \multicolumn{11}{|c|}{ Características de asociación y alianza: } \\
\hline Hypericum ericoides & 2.3 & 3.3 & 2.2 & 2.2 & 3.3 & 2.3 & 2.2 & 2.2 & 2.2 & 3.3 \\
\hline Chiliadenus glutinosus & 1.2 & 2.2 & 1.1 & 1.2 & 1.2 & 2.2 & 1.1 & 1.2 & 1.2 & 1.2 \\
\hline Helianthemum glabratum & + & + & +.2 & 1.1 & 1.1 & 1.1 & + & + & + & 1.1 \\
\hline Erica multiflora & +.2 & . & & + & +.2 & +.2 & + & + & + & + \\
\hline Ulex parviflorus & +.2 & . & +.2 & . & +.2 & +.2 & & + & . & + \\
\hline Polygala rupestris & +.2 & . & + & + & $\therefore$ & . & + & . & + & + \\
\hline Centaurea saguntina & . & + & . & . & 1.1 & . & + & + & . & +.2 \\
\hline Satureja innota & +.2 & & & + & + & + & . & . & + & . \\
\hline Antirrhinum litigiosum & + & + & . & + & + & . & . & & & + \\
\hline \multicolumn{11}{|l|}{ Diferenciales de variante: } \\
\hline Chamaerops humilis & . & . & . & . & . & . & +.2 & +.2 & +.2 & +.2 \\
\hline Asparagus horridus & . & . & . & . & . & . & + & + & + & + \\
\hline \multicolumn{11}{|l|}{ Características de orden y clase: } \\
\hline Fumana ericifolia & 1.1 & +.2 & 1.1 & 1.2 & 1.1 & +.2 & 1.2 & + & 1.2 & 1.2 \\
\hline Rosmarinus officinalis & +.2 & . & +.2 & 1.1 & +.2 & 1.1 & & + & + & + \\
\hline Fumana ericoides & + & . & . & + & + & + & +.2 & + & . & + \\
\hline Thymus vulgaris & +.2 & . & + & +.2 & . & + & + & . & . & + \\
\hline Globularia alypum & . & . & +.2 & + & . & +.2 & + & +.2 & . & + \\
\hline Coris fontqueri & +.2 & + & + & . & + & . & + & . & + & . \\
\hline Helianthemum syriacum & . & . & 1.1 & . & . & + & + & + & +.2 & \\
\hline Teucrium gracillimum & . & & + & . & + & + & . & + & & + \\
\hline Fumana thymifolia & . & +.2 & . & . & + & + & . & . & 1.1 & . \\
\hline Thesium divaricatum & + & . & . & + & . & + & . & . & + & . \\
\hline Phagnalon rupestre & . & . & + & . & + & . & . & + & + & . \\
\hline Asperula scabra & . & + & . & . & . & . & + & . & + & . \\
\hline Fumana laevipes & . & . & + & . & . & . & . & . & + & . \\
\hline Argyrolobium zanonii & . & . & & & . & + & . & . & . & + \\
\hline Bupleurum fruticescens & . & . & . & +.2 & . & . & . & . & . & . \\
\hline Fumana hispidula & . & . & . & $\cdot$ & . & . & . & . & +.2 & . \\
\hline \multicolumn{11}{|l|}{ Compañeras: } \\
\hline Sedum sediforme & . & +.2 & . & + & . & + & + & + & . & + \\
\hline Sedum album & +.2 & . & . & . & . & + & + & + & + & . \\
\hline Centaurium barrelieri & +.2 & . & . & + & + & . & . & . & + & . \\
\hline Hyparrhenia sinaica & + & . & . & + & + & . & . & . & + & . \\
\hline Stipa offneri & +.2 & . & + & . & + & . & . & . & . & . \\
\hline Brachypodium retusum & + & . & . & . & . & . & + & + & $\cdot$ & . \\
\hline Avenula bromoides & + & . & . & . & . & + & . & $\cdot$ & . & . \\
\hline Stipa parviflora & +.2 & . & +.2 & . & . & . & . & . & . & . \\
\hline Lapiedra martinezii & . & . & . & . & . & . & . & . & + & . \\
\hline Launaea pumila & . & . & . & . & . & . & . & . & + & . \\
\hline
\end{tabular}

Procedencia de los inventarios: 1.- Cs: Altura, pr. Loma Cabrera, YK1211. 2.- Cs: Segorbe, Barranco de la Jara, YK1900. 3.- Cs: Soneja, YK2010. 4.- Cs: Villatorcas, YK1812. 5.- V: Serra, pr. Rebalsadors, YJ1897. 6.- V: Casinos, hacia Alcublas, YJ0099. 7.- V: Sot de Ferrer, pr. El Picacho, YK2308. 8.- V: Segart, La Mola, YJ2494. 9.- V: La Pobla de Vallbona, pr. Torre de Portacoeli, YJ1390. 10.- V: Sagunto, cerro del Castillo Romano, YJ3395. 
Tabla 3.

Sideritido juryi-Hypericetum ericoidis M. B. Crespo, ass. nov.

(Hypericion ericoidis, Rosmarinetalia officinalis, Rosmarinetea)

\begin{tabular}{|c|c|c|c|c|c|c|c|c|c|c|}
\hline $\mathrm{N}^{\circ}$ de Orden & 1 & 2 & 3 & 4 & 5 & 6 & 7 & 8 & 9 & 10 \\
\hline Área $\left(m^{2}\right)$ & 20 & 10 & 15 & 25 & 20 & 20 & 15 & 20 & 25 & 10 \\
\hline Cobertura (\%) & 50 & 40 & 40 & 45 & 45 & 40 & 35 & 45 & 60 & 50 \\
\hline Inclinación $\left({ }^{\circ}\right)$ & $30 \mathrm{NW}$ & $15 \mathrm{E}$ & $30 N$ & $30 \mathrm{NW}$ & $5 \mathrm{~N}$ & $25 \mathrm{~S}$ & $40 \mathrm{~N}$ & $20 \mathrm{~N}$ & $5 \mathrm{~N}$ & $10 \mathrm{~S}$ \\
\hline Altitud (m.s.n.m.) & 200 & 250 & 100 & 100 & 450 & 100 & 100 & 180 & 300 & 280 \\
\hline Fecha (mes-año) & $8-91$ & $8-91$ & $7-92$ & $7-92$ & $8-92$ & $8-92$ & $8-91$ & $9-92$ & $7-92$ & $7-92$ \\
\hline \multicolumn{11}{|c|}{ Características de asociación y alianza: } \\
\hline Hypericum ericoides & 3.3 & 2.2 & 2.2 & 3.3 & 2.2 & 2.2 & 1.2 & 2.2 & 3.3 & 3.3 \\
\hline Chiliadenus glutinosus & 1.2 & +.2 & 1.1 & + & +.2 & 1.2 & 1.2 & 1.1 & + & + \\
\hline Helianthemum marifolium & +.2 & 1.2 & + & + & 1.1 & + & + & + & 1.1 & 1.1 \\
\hline Erica multiflora & + & + & +.2 & +.2 & $\therefore$ & + & $\therefore$ & 1.1 & 1.1 & +.2 \\
\hline Ulex parviflorus & 1.1 & + & . & $\cdot$ & 1.1 & . & 1.1 & + & + & + \\
\hline Sideritis juryi & 1.1 & + & . & + & + & + & . & + & . & + \\
\hline Polygala rupestris & . & +.2 & . & & . & + & + & . & . & + \\
\hline Teucrium edetanum & . & . & + & 1.2 & . & + & . & . & . & . \\
\hline \multicolumn{11}{|c|}{ Características de orden y clase: } \\
\hline Fumana ericifolia & +.2 & 1.1 & + & + & +.2 & 1.2 & 1.1 & +.2 & 1.2 & 1.2 \\
\hline Rosmarinus officinalis & 1.1 & + & & + & 1.1 & . & 1.1 & +.2 & +.2 & . \\
\hline Fumana ericoides & + & . & . & 1.1 & + & . & + & + & + & + \\
\hline Fumana laevipes & . & +.2 & + & + & . & + & + & . & + & + \\
\hline Fumana thymifolia & + & +.2 & + & + & . & . & + & . & . & + \\
\hline Phagnalon rupestre & + & + & . & . & + & + & . & + & + & . \\
\hline Thymus vulgaris & + & +.2 & + & . & . & . & . & + & + & . \\
\hline Teucrium gracillimum & + & + & . & . & & + & . & . & + & + \\
\hline Helianthemum syriacum & . & + & . & . & + & + & . & + & . & + \\
\hline Globularia alypum & . & $\cdot$ & + & . & + & . & . & + & . & $\cdot$ \\
\hline Atractylis humilis & . & +.2 & + & . & . & . & . & $\cdot$ & $\cdot$ & $\cdot$ \\
\hline Coris fontqueri & + & . & . & . & + & . & . & . & . & . \\
\hline Viola arborescens & . & + & . & . & . & . & . & . & . & + \\
\hline Argyrolobium zanonii & . & $\therefore$ & + & . & . & . & . & + & & . \\
\hline Ononis minutissima & . & 1.1 & . & . & . & . & . & . & & . \\
\hline \multicolumn{11}{|l|}{ Compañeras: } \\
\hline Sedum sediforme & +.2 & +.2 &. & 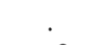 & + & + & + & . & +.2 & . \\
\hline Chamaerops humilis & . & . & +.2 & +.2 & . & +.2 & . & +.2 & . & . \\
\hline Avenula bromoides & + & + & . & . & + & + & . & . & . & . \\
\hline Brachypodium retusum & . & + & . & + & + & . & . & + & . & . \\
\hline Hyparrhenia sinaica & + & . & . & + & + & . & . & . & . & . \\
\hline Asparagus horridus & . & + & + & + & . & . & . & . & . & . \\
\hline Centaurium barrelieri & + & . & . & . & . & . & . & . & + . & \\
\hline Launaea pumila & . & . & + & . & . & + & . & . & . & . \\
\hline Stipa offneri & . & . & . & + & . & . & + & . & . & . \\
\hline Sedum album & + & . & & . & . & . & . & . & . & . \\
\hline Sedum acre & . & + & . & . & . & . & . & . & . & . \\
\hline
\end{tabular}

Procedencia de los inventarios:1.- V: Llíria, hacia Casinos, YJ0490. 2.- V: Llíria, Ermita San Miguel, YJ0688. 3.- V: Rocafort, pr. Campo Olivar, YJ2179. 4.- V: Godella, pr. Masía San Mauro, YJ2081. 5.- V: Casinos, pr. Mas de Somoza, XJ9395. 6.- V: Paterna, La Cañada, Martinot, YJ1478. 7.- V: Llíria, hacia Los Puchilis, YJ0790. 8.- V: Benaguacil, YJ0685. 9.- V: Llíria, pr. Cañada Parda, XJ9792. 10.- V: Llíria, pr. Mojón alto, XJ 9691. 
la Península Ibérica y De la Torre et al. (1996) para el sector Setabense.

Se reserva la categoría de subasociación para los sintáxones con una clara significación biogeográfica y la de variante para aquellos casos en los que la aparición de determinados táxones pone de manifiesto condiciones bioclimáticas o, en general, ecológicas, no ligadas a variaciones geográficas.

El tratamiento taxonómico aceptado sigue el criterio de Mateo y Crespo (1998). Cuando no se indica explícitamente, debe entenderse que los nombres citados corresponden a la subespecie o variedad típica. En las tablas figuran en todo caso los nombres abreviados correspondientes a la categoría infraespecífica aceptada.

\section{NOVEDADES SINTAXONÓMICAS}

\section{Teucrio edetani-Anthyllidetum terniflorae M.}

\section{B. Crespo, ass. nov.}

[Holotypus: Tabla 1, invent. 1.]

Combinación florística: matorral nanofanerofítico de talla media (hasta $1 \mathrm{~m}$ ) y cobertura generalmente elevada (más del 70\%), en la que dominan Anthyllis terniflora (Lag.) Pau y buen número de características de las unidades superiores (Erica multiflora L., Ulex parviflorus Pourr., etc.), dando un peculiar aspecto a esta asociación. Junto a ellos aparecen numerosos elementos termófilos o de matiz litoral, como Anthyllis cytisoides L., Viola arborescens L., Haplophyllum linifolium (L.) G. Don f. subsp. rosmarinifolium (Pers.) O. Bolòs y Vigo, Globularia alypum L., etc. Sin embargo, el hecho más notable es la presencia exclusiva de táxones de óptimo murcianoalmeriense como Onobrychis stenorhiza DC., Cistus heterophyllus Desf. subsp. carthaginensis (Pau) M. B. Crespo y Mateo, Lapiedra martinezii Lag. o Thymelaea argentata (Lam.) Pau, que junto con los endemismos edetanos Teucrium edetanum $\mathrm{M}$.
B. Crespo et al. y Sideritis juryi Peris et al., permiten reconocer fácilmente esta asociación frente a los sintáxones próximos. Es, quizás, la asociación de combinación florística más original y destacable en el seno de los Rosmarinion valencianos (tab. 4).

Ecología y bioclimatología: de óptimo fenológico primaveral, se instala sobre suelos calizos o calizo-margosos, que frecuentemente presentan una costra caliza ("tap") subsuperficial. Su óptimo se localiza en las áreas con bioclima termomediterráneo secosemiárido, desapareciendo rápidamente cuando el ombrotipo se hace francamente seco.

Fitogeografía: resulta un elemento endémico de los territorios edetanos orientales (gran parte de comarcas del Camp de Llíria y l'Horta Nord), en el extremo meridional del subsector Valenciano-Castellonense (sector Valenciano-Tarraconense, provincia CatalanoValenciano-Provenzal). La existencia de esta asociación endémica apoya el hecho de la independización corológica de lo que se ha venido llamando Zona o Distrito Edetánico (cf. Laguna et al., 1998).

Sinfitosociología: forma parte de la serie de los lentiscares seco-semiáridos (QuercoLentisceto Sigmetum).

Sintaxonomía: la ausencia de táxones de óptimo setabense, propios de los Teucrio latifolii-Thymenion piperellae (e.g., Teucrium homotrichum (Font Quer) Rivas Mart., Thymus piperella L., Satureja obovata Lag. subsp. valentina (G. López) M. B. Crespo, Helianthemum cinerum (Cav.) Pers subsp. rotundifolium (Dunal) Greuter y Burdet, Erica terminalis, Anthyllis onobrychioides, etc.) apunta hacia la inclusión de la nueva asociación en los Rosmarinenion, donde representa el extremo meridional de esta subalianza en tránsito hacia el dominio setabense.

Variabilidad: hacia los extremos de su área de distribución contacta con el resto de comunidades de la alianza, a través de diversas variantes, que suponen la transición hacia 
ombrotipos superiores:

a) variante típica de Teucrium edetanum (invs. 1-7), presente en las áreas centrales más secas del Camp de Llíria. Florísticamente queda caracterizada por la presencia de Cistus heterophyllus subsp. carthaginensis y Onobrychis stenorhiza. Con cierta frecuencia se presenta en su seno Helianthemum marifolium (L.) Mill. subsp. marifolium, sobre todo hacia el extremo occidental de su área, donde se establece el tránsito hacia la Helianthemo marifolii-Linetum suffruticosi sideritidetosum juryi.

b) variante de Helianthemum glabratum (invs. 8-9; Helianthemo glabratiGlobularietum alypum thymelaeetosum hirsutae Stübing et al. 1989, loc. cit.: 31, tab. 6, invent. 8), se observa en la vertiente sur de la Sierra Calderona y supone el contacto con la Helianthemo glabrati-Globularietum alypum Stübing et al. 1989, de distribución más septentrional. Florísticamente queda definida por la presencia de Helianthemum origanifolium (Lam.) Pers. subsp. glabratum (Willk.) Guinea y Heywood.

c) variante de Anthyllis lagascana (invent. 10), se presenta en el límite sudoeste de la asociación, marcando el contacto con la Hippocrepido scorpioidis-Anthyllidetum lagascanae. Florísticamente queda definida por Anthyllis lagascana Benedí (A. sericea Lag., non Willd.) y Thymus piperella.

Conservación: debido al intenso aprovechamiento urbanístico y agropecuario que sufre el territorio potencial de esta asociación, se ha constatado una alarmante pérdida de hábitat que podría llevar a su desaparición. Parece, pues, conveniente tomar medidas urgentes para la conservación de alguna de las mejores representaciones de esta asociación, que se encuentra claramente en peligro.

\section{Helianthemo glabrati-Hypericetum ericoidis}

M. B. Crespo, ass. nov.

[Holotypus: Tabla 2, invent. 5]
Combinación florística: brezal de roca de escasa talla y baja cobertura (menos del 50\%), dominado por Hypericum ericoides L., a la que acompaña buen número de características de las unidades superiores. La presencia de Helianthemum origanifolium subsp. glabratum, junto a endemismos de óptimo valencianotarraconense como Centaurea saguntina Mateo y M. B. Crespo (C. dufourii auct.) y Satureja innota (Pau) Pau ex Zapater, permite reconocer esta asociación frente a sus vicariantes territoriales. No obstante, está emparentada con la Helianthemo mollis-Hypericetum ericoidis R. Roselló 1994, propia de áreas espadánicas más húmedas y algo más frías (comarca del Alto Mijares, Castellón), diferenciándose por la ausencia de Helianthemum origanifolium subsp. molle (Cav.) Font Quer y Rothm., Galium maritimum L., Jasione mansanetiana R. Roselló y Peris o Phagnalon sordidum (L.) Rchb., entre otras (tab. 5).

Ecología y bioclimatología: se instala sobre lajas calizas o dolomíticas de escasa o nula inclinación. Su óptimo se localiza en las áreas con bioclimas termo- y mesomediterráneo seco.

Fitogeografía: resulta un elemento endémico de los territorios valencianocastellonenses centro-meridionales (sector Valenciano-Tarraconense, provincia CatalanoValenciano-Provenzal). Hacia el norte deja paso a su vicariante Helianthemo mollisHypericetum ericoidis, cuando el ombrotipo se hace seco-subhúmedo; hacia el sur lo hace con la Sideritido juryi-Hypericetum ericoidis, bajo ombrotipo seco-semiárido.

Sinfitosociología: se integra en la serie de los carrascales termófilos iberolevantinos (Rubio longifoliae-Querceto rotundifoliae Sigmetum). A menudo contacta topográficamente con matorrales de Helianthemo glabrati-Globularietum alypum (Rosmarinion), cuando el suelo gana potencia. Variabilidad: pueden reconocerse dos 


\begin{tabular}{lcccccccc}
\hline Número de orden & 1 & 2 & 3 & 4 & 5 & 6 & 7 & 8 \\
Número de inventarios & 10 & 8 & 6 & 7 & 11 & 10 & 12 & 10 \\
\hline
\end{tabular}

Características de las asociaciones:

Galium maritimum

Helianthemum molle

Helianthemum glabratum

Satureja gracilis

Anthyllis terniflora

Teucrium edetanum

Cistus carthaginensis

Onobrychis stenorrhiza

Anthyllis lagascana

Helianthemum rotundifolium

Características de las subalianzas:

Satureja innota

Biscutella carolipauana

Centaurea saguntina

Sideritis juryi

Thymus piperella

Teucrium homotrichum

Satureja valentina

Características de alianza:

Erica multiflora

Ulex parviflorus

Globularia alypum

Helianthemum marifolium

Anthyllis cytisoides

Thymelaea tinctoria

Cistus clusii

Sideritis tragoriganum

Thymelaea argentata

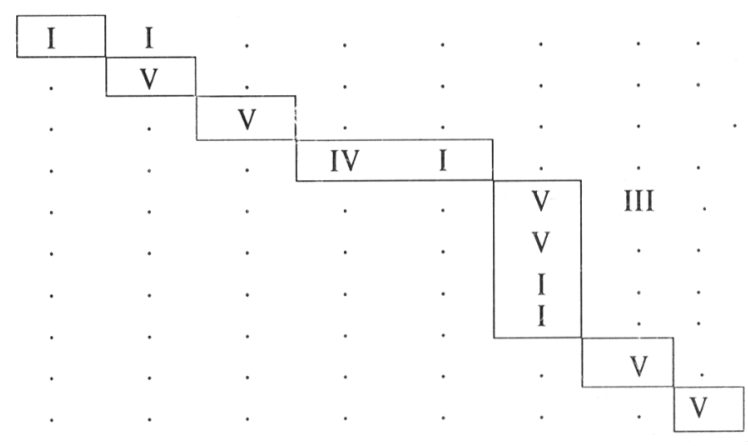

\begin{tabular}{|c|c|c|c|c|c|c|}
\hline II & I & . & . & . & . & . \\
\hline II & I & . & . & . & . & . \\
\hline I & I & & & . & . & . \\
\hline . & . & . & III & V & V & III \\
\hline$\cdot$ & & . & . & . & . & I \\
\hline . & . & . & . & . & . & . \\
\hline$\cdot$ & . & & . & . & . & . \\
\hline IV & V & V & V & $\mathrm{V}$ & $\mathrm{V}$ & V \\
\hline V & $\mathrm{V}$ & $\mathrm{V}$ & V & V & IV & V \\
\hline III & IV & V & III & IV & IV & V \\
\hline V & & . & V & $\mathrm{V}$ & III & V \\
\hline I & IV & III & I & III & . & . \\
\hline III & I & . & V & V & I & . \\
\hline III & . & I & V & III & II & IV \\
\hline II & II & II & . & . & . & . \\
\hline . & . & II & . & II & V & III \\
\hline
\end{tabular}

Procedencia de los inventarios:1.- Anthyllido-Cystetum clusii (Stübing et al., 1989; Phytocoenologia 17(1), tab. 4, invent. 1-10) 2.- Helianthemo mollis-Ulicetum parviflori (Stübing et al., loc. cit., tab. 5, invs. 1-8) 3.- Helianthemo glabrati-Globularietum alypum (Stübing et al., loc. cit., tab. 6, invs. 1-6) 4.Helianthemo marifolii-Linetum suffruticosi (Stübing et al., loc. cit., tab. 7, invs. 1-7) 5.- Helianthemo marifolii-Linetum suffruticosi sideritidetosum juryi (Stübing et al., loc. cit., tab. 8, invs. 1-9; tab. 7, invent. 8-9) 6.- Teucrio edetani-Anthyllidetum terniflorae (tab. 1) 7.- Hippocrepido scorpioidis-Anthyllidetum lagascanae (Stübing et al., loc. cit., tab. 9, invent. 1-12). 8.- Thymo piperellae-Helianthemetum marifolii (Stübing et al., loc. cit., tab. 10, invent. 1-10).

Tabla 4. Cuadro comparativo de las comunidades de Rosmarinion citadas en el texto (para más información sobre la alianza véase Stübing et al., 1989). 


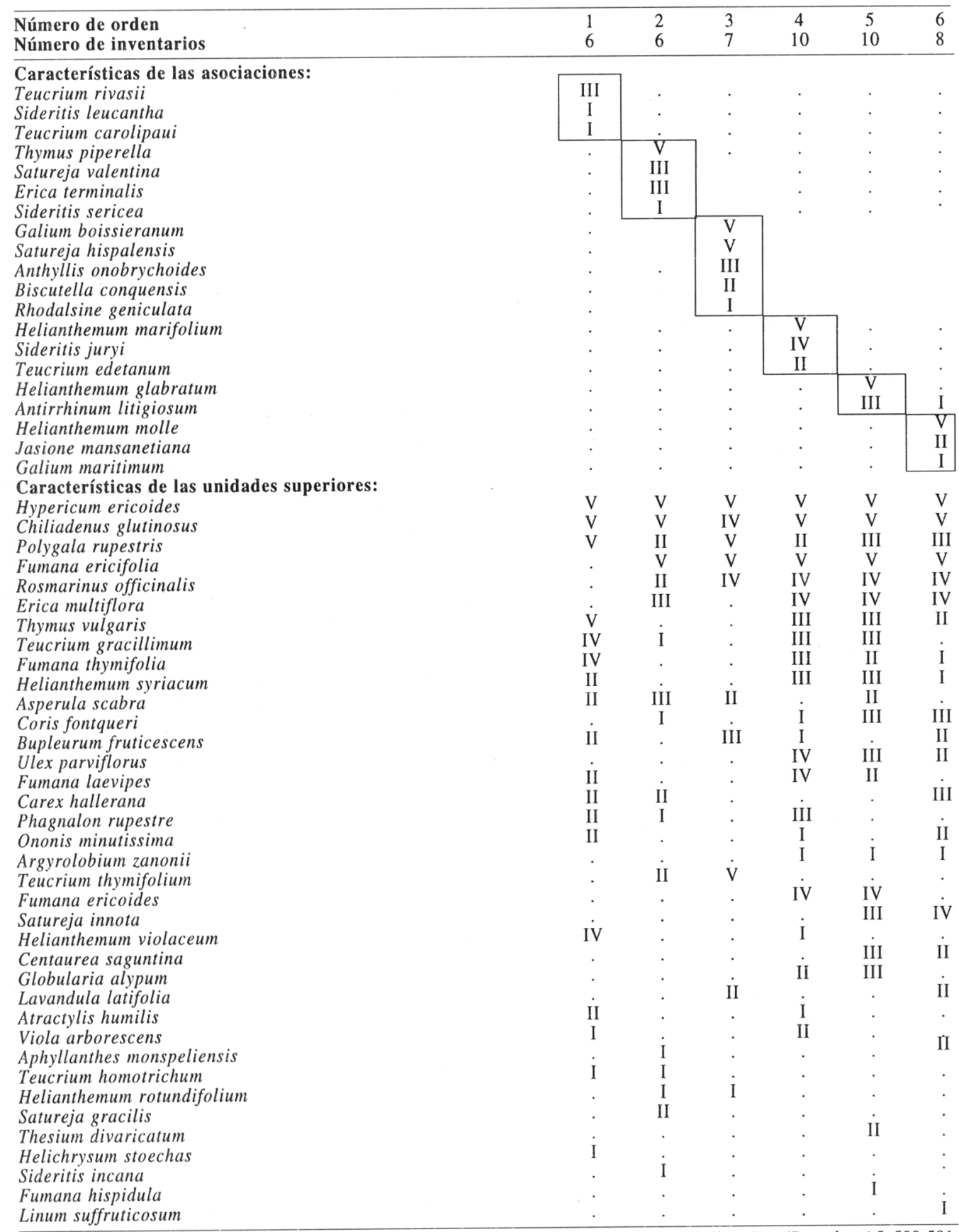

Procedencia de los inventarios: 1.- Fumano ericoidis-Hypericetum ericoidis O. Bolòs 1957 [Collect. Bot. (Barcelona) 5: 580-581, tab. 2, invent. 1-6]. 2.- Thymo piperellae-Hypericetum ericoidis M. Costa, Peris y Stübing in M. Costa y Peris 1985 [Lazaroa 6: 98 , tab. 6]. 3.- Galio boissierani-Hypericetum ericoidis Peris, Esteso y Stübing 1993 [Collect. Bot. (Barcelona) 22: 163, tab. 1]. 4.Sideritido juryi-Hypericetum ericoidis M. B. Crespo, ass. nov. (Tabla 2). 5.- Helianthemo glabrati-Hypericetum ericoidis M. B. Crespo, ass. nov. (tabla 3). 6.- Helianthemo mollis-Hypericetum ericoidis R. Roselló 1994 (loc. cit.: 533, tab. 71).

Tabla 5.- Cuadro comparativo de las comunidades de Hypericion ericoidis. 
variantes con valor termoclimático:

a) variante de Hypericum ericoides (invs. 1-6): la típica, propia de áreas mesomediterráneas, donde se encuentran ausentes elementos termófilos y se hace más patente la presencia de plantas como Satureja innota y Antirrhinum barrelieri Boreau subsp. litigiosum (Pau) O. Bolòs y Vigo.

b) variante de Chamaerops humilis (invs. 7-10): propia de territorios termomediterráneos. Florísticamente queda definida por la presencia de Chamaerops humilis L. y Asparagus horridus L. f.

Sideritido juryi-Hypericetum ericoidis M. B. Crespo, ass. nov.

[Holotypus: Tabla 3, invent. 1]

Combinación florística: brezal de roca de escasa talla y baja cobertura (menos del $40 \%$ ), dominado por Hypericum ericoides, a la que acompaña buen número de características de las unidades superiores. La presencia, aunque escasa y testimonial, de los endemismos edetanos Teucrium edetanum y Sideritis juryi, junto al elemento territorial Helianthemum marifolium subsp. marifolium, permite reconocer fácilmente esta asociación frente a los sintáxones próximos. La ausencia de plantas de óptimo espadánico, como Satureja innota, Centaurea saguntina o Helianthemum origanifolium (s.1.) la alejan de sus vicariantes septentrionales antes mencionadas, mientras que la ausencia de Thymus piperella, Teucrium homotrichum y Satureja obovata subsp. valentina la separan de la Thymo piperellaeHypericetum ericoidis M. Costa, Peris y Stübing in M. Costa y Peris 1985, su vicariante meridional setabense. El acusado carácter xérico de esta nueva asociación la hace converger con la Fumano ericoidisHypericetum ericoidis O. Bolòs 1957, de amplia distribución alicantina seco-semiárida; pero florísticamente las diferencias son muy notables (tab. 5).

Ecología y bioclimatología: se instala sobre lajas calizas o dolomíticas de escasa o nula inclinación. Su óptimo se localiza en las áreas con bioclimas termo- y mesomediterráneo seco-semiárido.

Fitogeografía: resulta un elemento endémico de los territorios valencianocastellonenses meridionales (sector Valenciano-Tarraconense, provincia CatalanoValenciano-Provenzal), ya en contacto con el dominio setabense. Hacia el norte, bajo ombrotipo seco, contacta con la Helianthemo glabrati-Hypericetum ericoidis estudiada anteriormente; hacia el sur deja paso a la Thymo piperellae-Hypericetum ericoidis, cuando el ombrotipo se hace seco-subhúmedo y la influencia setabense es palpable.

Sinfitosociología: forma parte de la serie de los lentiscares seco-semiáridos (QuercoLentisceto Sigmetum). Cuando el suelo gana potencia suele contactar topográficamente con matorrales de Rosmarinion: Teucrio edetaniAnthyllidetum terniflorae (en las áreas orientales de su distribución) o Helianthemo marifolii-Linetum suffruticosi sideritidetosum juryi (en las áreas occidentales).

\section{Hippocrepido scorpioidis-Anthyllidetum} lagascanae Stübing, Peris y M. Costa 1989 corr. M. B. Crespo hoc. loc.

[Holotypus: Stübing et al., Phytocoenologia 17(1): 38, tab. 9, invent. 3. 1989]

(Syn.: Hippocrepido comosaeAnthyllidetum sericeae Stübing et al. 1989 [Phytocoenologia 17(1): 36], nom. incorr. (Art. 43, 44))

Como ha demostrado Benedí (1995), el nombre Anthyllis sericea Lag. es ilegítimo por resultar homónimo posterior de $A$. sericea Willd., debiendo ser reemplazado por el nuevo nombre A. lagascana. Por esta razón, en virtud del Art. 44 no puede mantenerse el nombre original de esta asociación y se propone aquí corregirlo. Igualmente, cabe decir que tanto en 
la localidad clásica (La Cañada, Valencia), como en los territorios termo- y mesomediterráneos colindantes el taxon del género Hippocrepis que participa en los matorrales de Rosmarinion es Hippocrepis scorpioides Benth. (- H. glauca auct., non Ten.) y no $H$. comosa L., planta propia de pastizales mesofíticos de Festuco-Ononidetea striatae Rivas Mart. et al. 1991 que en la flora valenciana sólo se conoce de las altas montañas maestracenses (cf. Bolòs y Vigo, 1984: 645; Mateo y Crespo, 1998). Los materiales recolectados en la localidad clásica y áreas colindantes, que se conservan en $\mathrm{VAB}$ y $\mathrm{ABH}$, no dejan lugar a dudas sobre la filiación de dichas plantas. Según esto, el nombre de la asociación debe corregirse también en el sentido del Art. 43.

Se trata de una asociación muy localizada, endémica de La Cañada (Paterna, Valencia), donde se instala en las terrazas inferiores de la margen izquierda del río Turia, sobre suelos calizos de textura limoso-arenosa que retienen una notable humedad. Bioclimáticamente, dadas las peculiares preferencias ecológicas de la asociación, puede considerarse de óptimo termomediterráneo seco. Florísticamente queda bien definida por la presencia de Anthyllis lagascana junto con Helianthemum marifolium subsp. marifolium y Thymus piperella, aunque dado lo reducido del territorio que ocupa (no más de $3 \mathrm{~km}^{2}$ ) rápidamente entra en contacto y admite los elementos característicos de la Teucrio-Anthyllidetum terniflorae. Dinámicamente, forma parte de la serie de los lentiscares seco-semiáridos de QuercoLentisceto Sigmetum. Como en el caso de la Teucrio-Anthyllidetum se encuentra seriamente amenazada por la fuerte presión urbanística que sufre su exigua área potencial, por lo que deberían tomarse medidas para conservar sus mejores representaciones.

La ubicación sintaxonómica de la Hippocrepido-Anthyllidetum lagascanae resulta un tanto dificultosa, dado que en su seno participan el endemismo edetano Sideritis juryi (de Rosmarinenion) junto con Thymus piperella (de Teucrio-Thymenion piperellae); planta esta última que, según nuestras observaciones. se presenta de modo constante en la asociación contribuyendo a su caracterización, pese a lo que podría deducirse de la publicación original del sintaxon. Por ello, aunque podría argumentarse consistentemente la inclusión de esta asociación en cualquiera de ambas subalianzas, aceptamos la solución adoptada por Stübing et al. (1989), quienes la ubicaron en TeucrioThymenion piperellae. Además, ello se apoya en el hecho de que Anthyllis lagascana se comporta localmente en la Comunidad Valenciana como un elemento de distribución Setabense, en los subsectores AyoranoVillenense y Valenciano (cf. Serra y Crespo, 1998).

\section{Helianthemo marifolii-Linetum suffruticosi} Stübing, Peris y M. Costa 1989

[Phytocoenologia 17(1): 33] subass. linetosum suffruticosi

[Holotypus: Stübing et al., Phytocoenologia 17(1): 35, tab. 7, invent. 4. 1989]

La subasociación típica, cuyo óptimo se sitúa en territorios con bioclima mesomediterráneo (medio-superior) seco, de matiz continental, marcando el tránsito entre los Rosmarinion y los Sideritido-Salvion maestracenses. Florísticamente queda definida por la presencia de Helianthemum marifolium subsp. marifolium, Genista scorpius (L.) DC. y Satureja intricata Lange subsp. gracilis Rivas Mart. ex G. López, que aparecen junto a elementos de matiz litoral como Ulex parviflorus o Globularia alypum. Es importante indicar que la presencia de Sideritis juryi $(-S$. tragoriganum auct.; - S. saetabensis auct.) permite caracterizar territorialmente este sintaxon y no deja dudas sobre su inclusión en Rosmarinenion.

subass. sideritidetosum juryi Stübing, 
Peris y M. Costa 1989, corr. M. B. Crespo hoc. loc.

[Holotypus: Stübing et al., Phytocoenologia 17(1): 34, tab. 7, invent. 8. 1989]

(Syn.: Helianthemo marifolii-Linetum suffruticosi sideritetosum tragorigani Stübing et al. 1989 [Phytocoenologia 17(1): 35], nom. incorr. (Art. 43)]. Thymelaeetum tinctoriohirsutae Stübing, Peris y M. Costa 1989 [Phytocoenologia 17(1): 36, tab. 8, holotypus invent. 7])

Esta subasociación presenta su óptimo en las áreas con bioclima termomediterráneo seco o mesomediterráneo (inferior) seco, constituyendo el tránsito hacia otros matorrales edetanos más xerófilos. Florísticamente queda definida por la presencia de elementos termófilos como Anthyllis cytisoides, Thymelaea hirsuta (L.) Endl. o Viola arborescens (todas ellas de amplia distribución por el litoral iberolevantino), que actúan como diferenciales frente a la subasociación típica; así como por la escasez o ausencia de plantas de matiz más continental como Satureja intricata subsp. gracilis o Genista scorpius.

La asociación Thymelaeetum tinctoriohirsutae fue descrita de los territorios termo y mesomediterráneos situados en una estrecha franja colindante con la subasociación típica, aunque su área se amplió hasta zonas semiáridas de mayor carácter litoral sin aportar inventarios de tales territorios. Se trata de un sintaxon muy pobremente caracterizado, que consideramos más propio de los Rosmarinenion, como han indicado Díez Garretas et al. (1998), que de los Teucrio-Thymenion piperellae, donde fue inicialmente ubicado. En esta asociación, Thymelaea hirsuta resulta particularmente abundante. Esta planta muestra claras apetencias nitrófilas, por lo que a menudo interviene en comunidades de PeganoSalsoletea. Su participación en los matorrales seriales se reduce a las primeras fases de la colonización de terrenos baldíos o antiguos campos de cultivo, llegando a desaparecer una vez dichos matorrales alcanzan una mayor madurez. Dado que del estudio de las tablas originales de la Helianthemo marifolii-Linetum suffruticosi y la Thymelaeetum tinctoriohirsutae (tab. 4) no se deducen diferencias florísticas y corológicas suficientes como para permitir una cómoda separación, parece más apropiado considerarlas subasociaciones dentro de la primera, como aquí se propone. Es importante destacar que aunque en las tablas originales de Stübing et al. (1989) se hace referencia en el territorio de ambos sintáxones a Sideritis tragoriganum Lag. y S. saetabensis Rouy, actualmente podemos afirmar que el único taxon de este grupo presente en las áreas edetanas es $S$. juryi, por lo que en aplicación del Art. 43 se propone la corrección del nombre de la subasociación correspondiente.

\section{APÉNDICE FLORÍSTICO}

El grupo ibérico de Satureja obovata Lag. fue tratado muy acertadamente por López González (1982), quien lo subordinó a $S$. cuneifolia Ten. y reconoció en su seno cinco táxones de rango varietal. Posteriormente, Rivas Martínez (1982) realizó algunas precisiones, reivindicando la independencia del taxon ibérico ( $S$. obovata) del tirrénico $(S$. cuneifolia) y elevando la var. canescens Rouy, de distribución murciano-almeriense, al rango de subespecie. De modo similar, Cabezudo et al. (1991) trataron como subespecies las var. malacitana G. López (elemento de óptimo malacitano-axarquiense litoral) y var. hispalensis Pau (elemento de amplia distribución rondeña, subbética y almijarense). En el caso de los dos táxones iberolevantinos del agregado presentes en la flora valenciana, la var. valentina $\mathrm{G}$. López y la subsp. canescens (cf. Mateo y Crespo, 1998; Laguna et al., 1998) muestran suficientes diferencias morfológicas y biogeográficas como para tratarlar con igual rango taxonómico, por 10 
que parece apropiado establecer la siguiente combinación nomenclatural nueva:

Satureja obovata Lag. subsp. valentina ( $\mathrm{G}$. López) M. B. Crespo, comb. et stat. nov.

Basion.: S. cuneifolia subsp. obovata var. valentina G. López, Anales Jard. Bot. Madrid 38(2): 408. 1982.

Fitogeografía: endemismo de óptimo en el Sector Setabense (provincia CatalanoValenciano-Provenzal), en bioclimas termomesomediterráneo seco-subhúmedo. No obstante, existen unas polaciones aisladas en la comarca del Alto Mijares, Castellón (sector Valenciano-Tarraconense, provincia CatalanoValenciano-Provenzal), que merecen estudios particulares.

Fitosociología: Se presenta de ordinario en comunidades subrupícolas de Hypericion ericoidis, participando esporádicamente en comunidades de Asplenietalia petrarchae Br.B1. y Meier in Meier y Br.-BI. 1934 (Teucrion buxifolii Rivas Goday in Rivas Goday et al. 1955 y Asplenion petrarchae Br.-Bl. y Meier in Meier y Br.-B1. 1934) o de TeucrioThymenion piperellae (Rosmarinion).

Satureja obovata Lag. subsp.canescens (Rouy) Rivas Mart., Anales Edaf. Agrobiol. 41: 1515. 1982

Fitogeografía: endemismo de amplia distribución en la provincia MurcianoAlmeriense, con óptimo termomediterráneo semiárido (aunque puede alcanzar zonas mesomediterráneas localmente secas).

Fitosociología: Como el taxon anterior, suele presentarse en comunidades subrupícolas de Hypericion ericoidis, participando también esporádicamente en comunidades de Asplenietalia petrarchae (Teucrion buxifolii y Cosentinio-Lafuenteion Asensi et al., 1990) o de Anthyllidetalia terniflorae Rivas Goday et al. in Rivas Goday y Borja 1961.

\section{ESQUEMA SINTAXONÓMICO}

Cl. ROSMARINETEA OFFICINALIS Rivas Mart. et al. 1991

+ Ord. Rosmarinetalia Br.- Bl. ex Molinier 1934

* All. Rosmarinion Br.- Bl. ex Molinier 1934

- Suball. Rosmarinenion

Anthyllido cytisoidis-Cistetum clusii Br.B1. 1935, corr. O. Bolòs 1967. [Albaidarromerales termófilos, valenciano-tarraconenses septentrionales].

Helianthemo mollis-Ulicetum parviflorae Stübing, Peris y M. Costa 1989. [Aulagares termófilos, espadánicos septentrionales, secosubhúmedos].

Helianthemo glabrati-Globularietum alypum Stübing, Peris y M. Costa 1989 [Romeralaulagares termófilos, espadánicos meridionales, secos].

Helianthemo marifolii-Linetum suffruticosi Stübing, Peris y M. Costa 1989

subass. linetosum suffruticosi. [Romeralaulagares valenciano-castellonenses de matiz continental].

subass. sideritidetosum juryi Stübing, Peris y M. Costa 1989, corr. M. B. Crespo hoc. loc. (incl. Thymelaeetum tinctorio-hirsutae Stübing, Peris y M. Costa 1989). [Romeralaulagares valenciano-castellonenses de matiz sublitoral].

Teucrio edetani-Anthyllidetum terniflorae M. B. Crespo, ass. nov. [Brezal-albaidares termófilos, edetánicos, seco-semiáridos].

- Suball. Teucrio latifolii-Thymenion piperellae Stübing, Peris y M. Costa 1989

Thymo piperellae-Helianthemetum marifolii Rivas Goday 1958, corr. Díez Garretas, Fern. González y Asensi 1998. [Matorrales setabenses, seco-subhúmedos, con pebrella y zamarrilla de pastor].

Hippocrepido scorpioidis-Anthyllidetum lagascanae Stübing, Peris y M. Costa 1989, corr. M. B. Crespo hoc. loc. [Brezal-romerales termófilos, sin zamarrilla de pastor].

* All. Hypericion ericoidis Esteve ex M. Costa y Peris 1985

Thymo piperellae-Hypericetum ericoidis $\mathrm{M}$. 
Costa y Peris 1985. [Brezales de roca setabenses, seco-subhúmedos].

Helianthemo mollis-Hypericetum ericoidis R. Roselló 1994. [Brezales de roca espadánicos, seco-subhúmedos].

Helianthemo glabrati-Hypericetum ericoidis M. B. Crespo, ass. nov. [Brezales de roca espadánicos, secos].

Sideritido juryi-Hypericetum ericoidis M. B. Crespo, ass. nov. [Brezales de roca edetánicos, seco-semiáridos].

Galio boissierani-Hypericetum ericoidis Peris, Esteso y Stübing 1993. [Brezales de roca manchegos].

\section{BIBLIOGRAFÍA}

ALCARAZ, F. y A. DE LA TORRE -1988- Notas fitosociológicas sobre el sudeste ibérico. Acta Bot. Malacitana 13: 332-341.

BENEDÍ, C. -1995-Taxonomía del grupo de Anthyllis henoniana Coss. (Leguminosae): A. lagascana, nom. nov. Anales Jard. Bot. Madrid 53(2): 282284.

CABEZUDO, B., J. M. NIETO CALDERA y T. NAVARRO -1991- Catálogo de las labiadas (Labiatae) malacitanas (Málaga, España). Acta Bot. Malacitana 13: 332-341.

COSTA, M. y J. B. PERIS -1985- Aportación al conocimiento fitosociológico de las Sierras del Boquerón y Palomera (Valencia-Albacete): Los matorrales. Lazaroa 6: 81-103.

COSTA, M., J. B. PERIS y R. FIGUEROLA -1983Sobre los carrascales termomediterráneos valencianos. Lazaroa 4: 37-52.

CRESPO, M. B. -1989- Contribución al estudio florístico, fitosociológico y fitogeográfico de la Serra Calderona (Valencia-Castellón). Ser. Tesis Doct. 055-4 (microficha). Publ. Universidad de Valencia.

DE LA TORRE, A. y F. ALCARAZ-1994-Novedades sintaxonómicas en el orden Rosmarinetalia officinalis Br.-Bl. $1931 \mathrm{em} .1952$ para el sureste de España. Lazaroa 14: 125-138.

DE LA TORRE, A., F. ALCARAZ y M. B. CRESPO -1996- Aproximación a la biogeografía del sector Setabense (provincia Catalano-ValencianoProvenzal). Lazaroa 16: 141-158.

DÍEZ GARRETAS, B., A. ASENSI y F. FERNÁNDEZ GONZÁLEZ -1995- Revisión nomenclatural de la alianza Eryngio-Ulicion erinacei Rothm. 1943 (= Saturejo-Coridothymion capitati Rivas Goday \& Rivas Martínez 1969) y de sus sintáxones subordinados. Lazaroa 15: 218-225. Madrid.

DÍEZ GARRETAS, B., F. FERNÁNDEZ GONZÁLEZ y A. ASENSI -1998- Revisión nomenclatural de la clase Rosmarinetea officinalis en la Península Ibérica e Islas Baleares. Itinera Geobot. 11: 315364. León.

LAGUNA, E., M. B. CRESPO, G. MATEO, S. LÓPEZ UDIAS, C. FABREGAT, L. SERRA, J. J. HERRERO-BORGOÑÓN, J. L. CARRETERO, A. AGUILELLA, y R. FIGUEROLA-1998-Flora endémica, rara o amenazada de la Comunidad Valenciana. Colecc. Biodiversidad n ${ }^{\circ} 1$. Generalitat Valenciana.

LÓPEZ GONZÁLEZ, G. -1982- Conspectus Saturejarum ibericarum cum potioribus adnotationibus ad quasdam earum praesertim aspicientibus. Anales Jard. Bot. Madrid 38(2): 361-415.

MATEO, G. y M. B. CRESPO-1998-Manual para la determinación de la flora valenciana. Monogr. Fl. Montiber. 3. Valencia.

RIVAS MARTÍNEZ, S. -1982-Datos nomenclaturales y ecológicos sobre táxones españoles del género Satureja (Labiatae). Anales Edaf. Agrobiol. 41: 1513-1516.

RIVAS MARTÍNEZ, S. -1987-Memoria y Mapa de series de vegetación de España, escala 1:400.000. ICONA - M $^{\circ}$ de Agricultura, Pesca y Alimentación. Madrid.

RIVAS MARTÍNEZ, S. -1993- Bases para una nueva clasificación bioclimática de la Tierra. Folia Bot. Matritensis 10: 1-23.

RIVAS MARTÍNEZ, S. -1997- Syntaxonomical synopsis of the potential natural plant communities of North America, I. Itinera Geobot. 10: 5-148.

SERRA, L. y M. B. CRESPO -1998- Adiciones a la flora alicantina, III. Fl. Montiber. 9: 20-23.

STÜBING, G., J. B. PERIS y M. COSTA -1989-Los matorrales seriales termófilos valencianos. Phytocoenologia 17(1): 1-69. StuttgartBraunschweig.

Aceptado para su publicación en Octubre de 1999

Dirección del autor: Departamento de Ciencias Ambientales y Recursos Naturales (Botánica). Universidad de Alicante. Apdo. 99. E-03080 Alicante.e-mail: crespo@carn.ua.es 